\title{
THE MIXED HODGE-RIEMANN BILINEAR RELATIONS FOR COMPACT KÄHLER MANIFOLDS
}

\author{
TIEN-CUONG DINH AND VIÊT-ANH NGUYÊN
}

\begin{abstract}
Dedicated to Professor Henri Skoda on the occasion of his 60th birthday
\end{abstract}
\begin{abstract}
We prove the Hodge-Riemann bilinear relations, the hard Lefschetz theorem and the Lefschetz decomposition for compact Kähler manifolds in the mixed situation.
\end{abstract}

\section{Introduction AND STATEMENT OF THE MAIN RESUlts}

Around the year 1979, Khovanskii (see [8, 9, 10]) and Teissier (see [12, 13, 14]) discovered independently a beautiful intimate relationship between the theory of mixed volumes and algebraic geometry. In order to describe this connection we recall some facts from the theory of mixed volumes. Let $K_{1}, \ldots, K_{r}$ be $r n$-dimensional convex bodies in $\mathbb{R}^{n}$ and $I=\left(i_{1}, \ldots, i_{r}\right) \in \mathbb{N}^{r}$ with $|I|:=\sum_{s=1}^{r} i_{s}=n$. Then the (Minkowski) mixed volume $K^{I}=\left[K_{1}^{i_{1}} \ldots K_{r}^{i_{r}}\right]$ is determined by the folowing identity

$$
\mathrm{Vol}\left(\sum_{s=1}^{r} \lambda_{s} K_{s}\right)=\sum_{I=\left(i_{1}, \ldots, i_{r}\right):|I|=n} \frac{n !}{i_{1} ! \cdots i_{r} !} K^{I} \lambda_{1}^{i_{1}} \cdots \lambda_{r}^{i_{r}}, \quad \lambda_{1}, \ldots, \lambda_{r} \geq 0 .
$$

The Aleksandrov-Fenchel inequalities state that

$$
\left(\left[K_{1} K_{2} \ldots K_{r}\right]\right)^{2} \geq\left[K_{1} K_{1} K_{3} \ldots K_{r}\right] \cdot\left[K_{2} K_{2} K_{3} \ldots K_{r}\right]
$$

Now let $X$ be a complex algebraic manifold of dimension $n$ and $D_{1}, \ldots, D_{r}$ very ample divisors on $X$. Let $D^{I}=\left[D_{1}^{i_{1}} \ldots D_{r}^{i_{r}}\right]$ denote the index of intersection of $D_{1}^{i_{1}} \cap \cdots \cap D_{r}^{i_{r}}$, where $D_{s}^{r_{s}}$ stands for $D_{s} \cap \cdots \cap D_{s}$ ( $r_{s}$ times). Khovanskii and Teissier found out a profound analog between Aleksandrov-Fenchel inequalities and the Hodge-Riemann bilinear relations in algebraic geometry

$$
\left(\left[D_{1} D_{2} \ldots D_{r}\right]\right)^{2} \geq\left[D_{1} D_{1} D_{3} \ldots D_{r}\right] \cdot\left[D_{2} D_{2} D_{3} \ldots D_{r}\right] .
$$

Their proofs use the usual Hodge-Riemann bilinear relations (see Theorem 1.1 below) applied to Kähler forms corresponding to the divisors and an induction argument. Khovanskii and Teissier also noted that many other interesting inequalities from convex geometry (for example the Brunn-Minkowski inequality, Bonnesen-type inequalities etc.) either could be deduced from the Hodge-Riemann bilinear relations, or find their analogs for algebraic varieties that generalize the Hodge-Riemann

2000 Mathematics Subject Classification. Primary 32Q15, Secondary 58A14, 14Fxx.

Key words and phrases. compact Kähler manifold, Hodge theory, mixed volume. 
bilinear relations. Based on this point of view P. McMullen (see [11]) developed a deep and important generalization of Aleksandrov-Fenchel inequalities for simple convex polytopes. On the other hand, Khovanskii and Teissier's discovery also suggests a generalization of the mixed Hodge-Riemann bilinear relations in the context of compact Kähler manifolds. That is the main motivation of our work.

Let $X$ be a compact Kähler manifold of dimension $n$. Let $0 \leq p, q \leq n$ and $0 \leq r \leq 2 n$ be integers. One denotes by $\mathcal{E}^{p, q}(X)$ (resp. $L_{p, q}^{2}(X)$ ) the space of complex-valued differential forms of bidegree $(p, q)$ on $X$ with smooth coefficients (resp. with $L^{2}$-coefficients). For $\alpha \in L_{p, q}^{2}(X),\|\alpha\|_{L^{2}}$ denotes its $L^{2}$-norm, i.e. the sum of $L^{2}$-norms of its coefficients on charts. In the sequel $\mathcal{H}^{p, q}(X)$ denote the space of smooth d-closed $(p, q)$-forms modulo smooth d-exact $(p, q)$-forms. Moreover, for any smooth d-closed form $\alpha \in \mathcal{E}^{p, q}(X),[\alpha]$ denotes the class of $\alpha$ in $\mathcal{H}^{p, q}(X)$. We can identify $\mathcal{H}^{p, q}(X)$ to the subspace of $\mathcal{H}^{p+q}(X)$ spanned by classes of smooth d-closed $(p, q)$-forms. The classical Hodge decomposition theorem asserts that

$$
\mathcal{H}^{r}(X)=\bigoplus_{p+q=r} \mathcal{H}^{p, q}(X) \quad \text { and } \quad \mathcal{H}^{p, q}(X)=\overline{\mathcal{H}^{q, p}(X)}
$$

We refer the reader to [2, 6, 16, 17] for the basics of the Hodge theory and to [1, 7, 10, 13, 15, for some of its advanced aspects.

Fix non-negative integers $p, q$ such that $p+q \leq n$. Let $\omega_{1}, \ldots, \omega_{n-p-q+1}$ be Kähler forms. Put $\Omega:=\omega_{1} \wedge \cdots \wedge \omega_{n-p-q}$. Consider the mixed primitive subspace

$$
P^{p, q}(X):=\left\{[\alpha] \in \mathcal{H}^{p, q}(X):[\alpha] \wedge[\Omega] \wedge\left[\omega_{n-p-q+1}\right]=0\right\} .
$$

Let us define the mixed Hodge-Riemann bilinear form on $\mathcal{H}^{p, q}(X) \otimes \mathcal{H}^{p, q}(X)$ as follows

$$
Q([\alpha],[\beta]):=i^{p-q}(-1)^{\frac{(n-p-q)(n-p-q-1)}{2}} \int_{X} \alpha \wedge \bar{\beta} \wedge \Omega .
$$

Observe that $Q(\cdot, \cdot)$ is a sesquilinear Hermitian symmetric form.

The classical Hodge-Riemann bilinear relations state that

Theorem 1.1. If $\omega_{1}=\cdots=\omega_{n-p-q+1}$, then $Q(\cdot, \cdot)$ is positive definite on the primitive space $P^{p, q}(X)$.

The open question can be formulated as follows:

Does Theorem 1.1 still hold if $\omega_{1}, \ldots, \omega_{n-p-q+1}$ are arbitrary Kähler forms?

An attempt towards this generalization is made by Gromov. Namely, the following theorem is stated in [7].

Theorem 1.2. (Gromov's Theorem) If $p=q$, then $Q(\cdot, \cdot)$ is positive semi-definite on $P^{p, q}(X)$, that is, $Q([\alpha],[\alpha]) \geq 0$ for $\alpha \in P^{p, q}(X)$.

However, Gromov only gave therein a complete proof for the special case where $p=q=1$. On continuation of Gromov's work and using Aleksandrov's approach, Timorin has proved a general mixed Hodge-Riemann bilinear relations, but only in the linear situation [15] (see also [10, 13]). His result may be rephrased as follows (see also Proposition 2.1 below). 
Theorem 1.3. (Timorin's Theorem) If $X$ is a complex torus of dimension $n$, then $Q(\cdot, \cdot)$ is positive definite on $P^{p, q}(X)$.

The purpose of this article is to prove the above theorems in the general context. Now we state the main results.

Theorem A. Let $X$ be a compact Kähler manifold of dimension $n$ and $p, q$ integers such that $0 \leq p, q \leq p+q \leq n$. Then, for arbitrary Kähler forms $\omega_{1}, \ldots, \omega_{n-p-q+1}$, the mixed Hodge-Riemann bilinear form $Q(\cdot, \cdot)$ is positive definite on the mixed primitive subspace $P^{p, q}(X)$.

Note that when $\omega_{j}$ are cohomologous to very ample divisors of $X$, by Bertini theorem, one can replace $\left[\omega_{j}\right]$ by divisors $D_{j}$ which intersects transversally. Then one deduces from the classical Hodge-Riemann theorem on the submanifold $D:=$ $D_{1} \cap \cdots \cap D_{n-p-q}$ that $Q([\alpha],[\alpha]) \geq 0$ for all $[\alpha]$ satisfying $[\alpha] \wedge\left[\omega_{n-p-q+1}\right]=0$ on $\mathcal{H}^{p+1, q+1}(D)$ (see also [8, 9, 13, and [16]). This is the original reason to believe that the mixed Hodge-Riemann bilinear relations hold in the general situation.

The following results generalize the hard Lefschetz theorem and the Lefschetz decomposition theorem.

Theorem B. Let $X$ be a compact Kähler manifold of dimension $n$ and $p, q$ integers such that $0 \leq p, q \leq p+q \leq n$. Then, for arbitrary Kähler forms $\omega_{1}, \ldots, \omega_{n-p-q}$, the linear map $\tau: \mathcal{H}^{p, q}(X) \longrightarrow \mathcal{H}^{n-q, n-p}(X)$ given by

$$
\tau([\alpha]):=[\Omega] \wedge[\alpha], \quad[\alpha] \in \mathcal{H}^{p, q}(X),
$$

where $[\Omega]:=\left[\omega_{1}\right] \wedge \cdots \wedge\left[\omega_{n-p-q}\right]$, is an isomorphism.

Theorem C. Let $X$ be a compact Kähler manifold of dimension $n$ and $p, q$ integers such that $0 \leq p, q \leq p+q \leq n$. Then, for arbitrary Kähler forms $\omega_{1}, \ldots, \omega_{n-p-q+1}$, the following canonical decomposition holds

$$
\mathcal{H}^{p, q}(X)=P^{p, q}(X) \oplus\left[\omega_{n-p-q+1}\right] \wedge \mathcal{H}^{p-1, q-1}(X),
$$

with the convention that $\mathcal{H}^{p-1, q-1}(X):=0$ if either $p=0$ or $q=0$.

We close the introduction with a brief outline of the paper to follow.

Our strategy is to reduce the general case to the linear case. In order to achieve this reduction we apply the $L^{2}$-technique to solve a $\mathrm{dd}^{\mathrm{c}}$-equation. Recall here that $\mathrm{d}=\partial+\bar{\partial}, \mathrm{d}^{\mathrm{c}}=\frac{i}{2 \pi}(\bar{\partial}-\partial)$ and $\mathrm{dd}^{\mathrm{c}}=\frac{i}{\pi} \partial \bar{\partial}$. Section 2 is then devoted to developing the necessary technique. We begin this section by collecting some results of Timorin and by establishing some estimates. This will enable us to construct a solution of the above equation. We will, in the remaining part of Section 2, regularize this solution. Based on the results of Section 2, the proofs of the main theorems are presented in Section 3.

The mixed Hodge-Riemann theorem is not true in general if we replace $[\Omega]$ by the class of a smooth strictly positive form as a simple example in [1] shows. However, by continuity, it holds for every class $[\Omega]$ close enough to a product of Kähler classes. In Section 4 we describe the domain of validity of this theorem in the case where $p=q=1$. 
Acknowledgment. We would like to thank the referee for many interesting suggestions and remarks. We are also grateful to Professor Nessim Sibony for very stimulating discussions. The second author wishes to express his gratitude to the Max-Planck Institut für Mathematik in Bonn (Germany) for its hospitality and its support.

\section{Preparatory Results}

In the first two propositions we place ourselves in the linear context. For $0 \leq p, q \leq$ $n$, let $\Lambda^{p, q}\left(\mathbb{C}^{n}\right)$ denote the space of $(p, q)$-forms with complex-constant coefficients. $\Lambda^{p, q}\left(\mathbb{C}^{n}\right)$ is equipped with the Euclidean norm $\|\cdot\|$. We first recall Timorin's result [15].

Proposition 2.1. Let $p, q$ be integers such that $0 \leq p, q \leq p+q \leq n$ and $\omega_{1}, \ldots, \omega_{n-p-q+1}$ strictly positive forms of $\Lambda^{1,1}\left(\mathbb{C}^{n}\right)$. Define the sesquilinear Hermitian symmetric form

$$
Q(\alpha, \beta):=i^{p-q}(-1)^{\frac{(n-p-q)(n-p-q-1)}{2}} *(\alpha \wedge \bar{\beta} \wedge \Omega), \quad \alpha, \beta \in \Lambda^{p, q}\left(\mathbb{C}^{n}\right),
$$

where $*$ is the Hodge star operator, and $\Omega:=\omega_{1} \wedge \cdots \wedge \omega_{n-p-q}$. Define the mixed primitive subspace

$$
P^{p, q}\left(\mathbb{C}^{n}\right):=\left\{\alpha \in \Lambda^{p, q}\left(\mathbb{C}^{n}\right): \alpha \wedge \Omega \wedge \omega_{n-p-q+1}=0\right\}
$$

Then

(a) The operator of multiplication by $\Omega$ induces an isomorphism between $\Lambda^{p, q}\left(\mathbb{C}^{n}\right)$ and $\Lambda^{n-q, n-p}\left(\mathbb{C}^{n}\right)$.

(b) $Q(\cdot, \cdot)$ is positive definite on $P^{p, q}\left(\mathbb{C}^{n}\right)$.

(c) The space $\Lambda^{p, q}\left(\mathbb{C}^{n}\right)$ splits into the $Q$-orthogonal direct sum

$$
\Lambda^{p, q}\left(\mathbb{C}^{n}\right)=P^{p, q}\left(\mathbb{C}^{n}\right) \oplus \omega_{n-p-q+1} \wedge \Lambda^{p-1, q-1}\left(\mathbb{C}^{n}\right),
$$

with the convention that $\Lambda^{p-1, q-1}\left(\mathbb{C}^{n}\right):=0$ if either $p=0$ or $q=0$.

Proof. See Proposition 1, the Main Theorem and Corollary 2 in [15].

The following estimate will be crucial later on.

Proposition 2.2. There are finite positive constants $C_{1}$ and $C_{2}$ such that

$$
C_{1} \cdot\left\|\alpha \wedge \Omega \wedge \omega_{n-p-q+1}\right\|^{2}+C_{2} \cdot \Re Q(\alpha, \alpha) \geq\|\alpha\|^{2}
$$

for all forms $\alpha \in \Lambda^{p, q}\left(\mathbb{C}^{n}\right)$.

Proof. By Proposition 2.1(a) we may find a positive finite constant $C$ so that

$$
\frac{\|\gamma\|}{C} \leq\left\|\gamma \wedge \Omega \wedge \omega_{n-p-q+1}^{2}\right\| \leq C \cdot\|\gamma\|, \quad \gamma \in \Lambda^{p-1, q-1}\left(\mathbb{C}^{n}\right) .
$$

By Proposition 2.11(c) we may write

$$
\alpha=\beta+\omega_{n-p-q+1} \wedge \gamma, \quad \beta \in P^{p, q}\left(\mathbb{C}^{n}\right), \gamma \in \Lambda^{p-1, q-1}\left(\mathbb{C}^{n}\right) .
$$

Then we have

$$
Q(\alpha, \alpha)=Q(\beta, \beta)+Q\left(\omega_{n-p-q+1} \wedge \gamma, \omega_{n-p-q+1} \wedge \gamma\right)
$$


On the other hand, since $\beta \in P^{p, q}\left(\mathbb{C}^{n}\right)$, one gets that

$$
\left\|\alpha \wedge \Omega \wedge \omega_{n-p-q+1}\right\|=\left\|\gamma \wedge \Omega \wedge \omega_{n-p-q+1}^{2}\right\| \geq \frac{\|\gamma\|}{C}
$$

where the estimate follows from the left-side estimate in (2.1). Therefore, we obtain, for $C^{\prime}>0$ large enough,

$$
\|\alpha\|^{2} \leq C^{\prime}\left(\|\beta\|^{2}+\|\gamma\|^{2}\right) \leq C^{\prime}\|\beta\|^{2}+C^{\prime} C^{2}\left\|\alpha \wedge \Omega \wedge \omega_{n-p-q+1}\right\|^{2} .
$$

On the other hand, by Proposition 2.1(b) we may find a positive finite constant $C^{\prime \prime}$ so that

$$
\begin{aligned}
\|\beta\|^{2} \leq C^{\prime \prime} \cdot Q(\beta, \beta) & =C^{\prime \prime} \cdot\left(Q(\alpha, \alpha)-Q\left(\omega_{n-p-q+1} \wedge \gamma, \omega_{n-p-q+1} \wedge \gamma\right)\right) \\
& =C^{\prime \prime} \cdot\left(\Re Q(\alpha, \alpha)-\Re Q\left(\omega_{n-p-q+1} \wedge \gamma, \omega_{n-p-q+1} \wedge \gamma\right)\right) \\
& \leq C^{\prime \prime} \cdot \Re Q(\alpha, \alpha)+C^{\prime \prime} C^{2} \cdot\|\gamma\|^{2} \\
& \leq C^{\prime \prime} \cdot \Re Q(\alpha, \alpha)+C^{\prime \prime} C^{4} \cdot\left\|\alpha \wedge \Omega \wedge \omega_{n-p-q+1}\right\|^{2},
\end{aligned}
$$

where the first identity follows from (2.2), the second estimate from the right-side estimate in (2.1), and the last one from (2.3). This, combined with (2.4), implies the desired estimate for $C_{1}:=C^{\prime} C^{\prime \prime}$ and $C_{2}:=C^{\prime} C^{\prime \prime} C^{4}+C^{\prime} C^{2}$.

Proposition 2.3. We keep the hypothesis and the notation in the statement of Theorem A. Assume that $p \geq 1$ and $q \geq 1$. Then, for every d-closed form $f \in$ $\mathcal{E}^{p, q}(X)$ such that $[f] \in P^{p, q}(X)$, there is a form $u \in L_{p-1, q-1}^{2}(X)$ such that

$$
\operatorname{dd}^{\mathrm{c}} u \wedge \Omega \wedge \omega_{n-p-q+1}=f \wedge \Omega \wedge \omega_{n-p-q+1} .
$$

Proof. Consider the subspace $H$ of $L_{n-p+1, n-q+1}^{2}(X)$ defined by

$$
H:=\left\{\operatorname{dd}^{\mathrm{c}} \alpha \wedge \Omega \wedge \omega_{n-p-q+1}: \alpha \in \mathcal{E}^{q-1, p-1}(X)\right\} .
$$

We construct a linear form $h$ on $H$ as follows

$$
h\left(\operatorname{dd}^{\mathrm{c}} \alpha \wedge \Omega \wedge \omega_{n-p-q+1}\right):=(-1)^{p+q} \int_{X} \alpha \wedge f \wedge \Omega \wedge \omega_{n-p-q+1} .
$$

We now check that $h$ is a well-defined bounded linear form with respect to the $L^{2}$-norm restricted to $H$. To this end one first shows that there is a positive finite constant $C$ such that

$$
\left\|\mathrm{dd}^{\mathrm{c}} \alpha\right\|_{L^{2}} \leq C \cdot\left\|\mathrm{dd}^{\mathrm{c}} \alpha \wedge \Omega \wedge \omega_{n-p-q+1}\right\|_{L^{2}} .
$$

To prove (2.6) we first use a compactness argument to find finite disjoint open sets $\left(U_{j}\right)_{j=1}^{N}$ of $X$ so that $\overline{U_{j}}$ is contained in a local chart, and that $\partial U_{j}$ is piecewisely smooth, and that $X=\bigcup_{j=1}^{N} \overline{U_{j}}$. One next invokes the estimate in Proposition 2.2 for every point in each $U_{j}, j=1, \ldots, N$. Then one integrates this estimate over $X$. We extend the bilinear form $Q(\cdot, \cdot)$ given by formula (1.2) in a canonical way to $\mathcal{E}^{p, q}(X) \otimes \mathcal{E}^{p, q}(X)$ :

$$
Q(\alpha, \beta):=i^{p-q}(-1)^{\frac{(n-p-q)(n-p-q-1)}{2}} \int_{X} \alpha \wedge \bar{\beta} \wedge \Omega, \quad \alpha, \beta \in \mathcal{E}^{p, q}(X) .
$$


Consequently, for suitable positive finite constants $C$ and $C^{\prime}$,

$$
\left\|\mathrm{dd}^{\mathrm{c}} \alpha\right\|_{L^{2}}^{2} \leq C \cdot\left\|\mathrm{dd}^{\mathrm{c}} \alpha \wedge \Omega \wedge \omega_{n-p-q+1}\right\|_{L^{2}}^{2}+C^{\prime} \cdot \Re Q\left(\operatorname{dd}^{\mathrm{c}} \alpha, \operatorname{dd}^{\mathrm{c}} \alpha\right) .
$$

On the other hand, applying Stokes' Theorem yields that

$$
Q\left(\operatorname{dd}^{\mathrm{c}} \alpha, \operatorname{dd}^{\mathrm{c}} \alpha\right)=i^{p-q}(-1)^{\frac{(n-p-q)(n-p-q-1)}{2}} \int_{X} \operatorname{dd}^{\mathrm{c}} \alpha \wedge \operatorname{dd}^{\mathrm{c}} \bar{\alpha} \wedge \Omega=0 .
$$

This, combined with (2.7), implies (2.6).

By hypothesis the smooth form $f \wedge \Omega \wedge \omega_{n-p-q+1}$ is d-exact. Consequently, it follows from [2, p. 41] that there is a form $g \in \mathcal{E}^{n-q, n-p}(X)$ such that

$$
\operatorname{dd}^{\mathrm{c}} g=f \wedge \Omega \wedge \omega_{n-p-q+1} .
$$

Applying Stokes' Theorem, we obtain that

$$
\begin{aligned}
\left|\int_{X} \alpha \wedge f \wedge \Omega \wedge \omega_{n-p-q+1}\right| & =\left|\int_{X} \alpha \wedge \mathrm{dd}^{\mathrm{c}} g\right|=\left|\int_{X} \mathrm{dd}^{\mathrm{c}} \alpha \wedge g\right| \\
& \leq\|g\|_{L^{2}} \cdot\left\|\mathrm{dd}^{\mathrm{c}} \alpha\right\|_{L^{2}} \\
& \leq C\|g\|_{L^{2}} \cdot\left\|\mathrm{dd}^{\mathrm{c}} \alpha \wedge \Omega \wedge \omega_{n-p-q+1}\right\|_{L^{2}}
\end{aligned}
$$

where the latter estimate follows from (2.6). In particular, we have

$$
\int_{X} \alpha \wedge f \wedge \Omega \wedge \omega_{n-p-q+1}=0 \quad \text { when } \quad \operatorname{dd}^{\mathrm{c}} \alpha \wedge \Omega \wedge \omega_{n-p-q+1}=0 .
$$

In summary, we have just shown that $h$ given by (2.5) is a well-defined bounded linear form with respect to the $L^{2}$-norm restricted to $H$, and its norm is dominated by $C\|g\|_{L^{2}}$. Applying the Hahn-Banach Theorem, we may extend $h$ to a bounded linear form on $L_{n-p+1, n-q+1}^{2}(X)$. Let $u$ be a form in $L_{p-1, q-1}^{2}(X)$ that represents $h$. Then, in virtue of (2.5), we have that

$$
\int_{X} u \wedge \operatorname{dd}^{\mathrm{c}} \alpha \wedge \Omega \wedge \omega_{n-p-q+1}=(-1)^{p+q} \int_{X} \alpha \wedge f \wedge \Omega \wedge \omega_{n-p-q+1}
$$

for all test forms $\alpha \in \mathcal{E}^{q-1, p-1}(X)$. This is the desired identity of the proposition.

We need to regularize the solution $u$ given by the previous proposition. This is the purpose of the following result.

Proposition 2.4. We keep the hypothesis and the conclusion in the statement of Proposition 2.3. Then, there is a form $v \in \mathcal{E}^{p-1, q-1}(X)$ such that $\mathrm{dd}^{\mathrm{c}} v=\mathrm{dd}^{\mathrm{c}} u$.

Proof. First we like to equip the vector bundle $\mathcal{E}^{p, q}(X)$ with a special Hermitian metric. To this end suppose without loss of generality that $p \leq q$. For any $\alpha \in$ $\mathcal{E}^{p, q}(X)$, we apply Proposition [2.1(c) repeatedly in order to obtain the following unique decomposition

$$
\alpha=\sum_{j=0}^{p} \alpha_{j} \wedge \omega_{n-p-q+1}^{p-j}
$$


where $\alpha_{j} \in \mathcal{E}^{j, q-p+j}(X)$ such that $\alpha_{j} \wedge \Omega \wedge \omega_{n-p-q+1}^{2 p-2 j+1}=0$ (see also (1.1)). Now we can define a new form $\widetilde{\alpha} \in \mathcal{E}^{p, q}(X)$ as follows

$$
\widetilde{\alpha}:=\sum_{j=0}^{p}(-1)^{p-j} \alpha_{j} \wedge \omega_{n-p-q+1}^{p-j} .
$$

Define an inner product $\langle\cdot, \cdot\rangle$ on $\mathcal{E}^{p, q}(X)$ by setting

$$
\langle\alpha, \beta\rangle:=Q(\alpha, \widetilde{\beta}), \quad \alpha, \beta \in \mathcal{E}^{p, q}(X),
$$

where $Q(\cdot, \cdot)$ is given by the same integral as in (1.2). Using Proposition 2.1 (c), one may rewrite (2.10) as follows

$$
\langle\alpha, \beta\rangle=\sum_{j=0}^{p}(-1)^{p-j} Q\left(\omega_{n-p-q+1}^{p-j} \wedge \alpha_{j}, \omega_{n-p-q+1}^{p-j} \wedge \beta_{j}\right),
$$

where the $\beta_{j}$ 's are determined by $\beta$ in virtue of (2.8). Applying Proposition 2.1(b) and using (1.2) and (2.8)-(2.10), one can check that $\langle\cdot, \cdot\rangle$ defines a Hermitian metric on $\mathcal{E}^{p, q}(X)$. Moreover, if we consider the norm $\|\alpha\|:=\sqrt{\langle\alpha, \alpha\rangle}$, then there is a positive finite constant $C$ such that

$$
\frac{1}{C} \cdot\left(\sum_{j=0}^{p}\left\|\alpha_{j}\right\|_{L^{2}}\right) \leq\|\alpha\| \leq C \cdot \sum_{j=0}^{p}\left\|\alpha_{j}\right\|_{L^{2}}
$$

Consider the following form of bidegree $(p, q)$

$$
h:=\operatorname{dd}^{\mathrm{c}} u-f .
$$

Then in virtue of Proposition 2.3 and of the hypothesis, $h$ belongs to the Sobolev space $W^{-2}\left(\mathcal{E}^{p, q}(X)\right)^{1}$. In addition, the following identities hold

$$
\bar{\partial} h=0, \quad \partial h=0 \quad \text { and } \quad h \wedge \Omega \wedge \omega_{n-p-q+1}=0 .
$$

For any form $\alpha \in \mathcal{E}^{p, q-1}(X)$, we have that

$$
\langle\bar{\partial} \alpha, h\rangle=Q(\bar{\partial} \alpha, h)=i^{p-q}(-1)^{p+q-1+\frac{(n-p-q)(n-p-q-1)}{2}} \int_{X} \alpha \wedge \overline{\partial h} \wedge \Omega=0,
$$

where the first identity follows from (2.8)-(2.10) and from the third identity in (2.12), the second one from (1.2) and from an application of Stokes' Theorem, and the last one from the second identity in (2.12). Let $\bar{\partial}^{*}$ be the adjoint of $\bar{\partial}$ with respect to the inner product given in (2.10). Then we have shown that $\bar{\partial}^{*} h=0$. On the other hand, $\bar{\partial} h=0$ by (2.12) and $h \in W^{-2}\left(\mathcal{E}^{p, q}(X)\right)$. Therefore, $h$ is a harmonic current with respect to the Laplacian operator $\overline{\partial \partial}^{*}+\bar{\partial}^{*} \bar{\partial}$ (see Section 5 in 17, Chap. IV]). Consequently, by elliptic regularity (see Theorem 4.9 in [17, Chap. IV]) $h$ is smooth. Hence, $\mathrm{dd}^{\mathrm{c}} u$ is smooth by (2.11). By the classical Hodge theory [2, p. 41] there is a $v \in \mathcal{E}^{p-1, q-1}(X)$ such that $\operatorname{dd}^{\mathrm{c}} v=\mathrm{dd}^{\mathrm{c}} u$. Hence, the proof is finished.

\footnotetext{
${ }^{1}$ For the Sobolev spaces on compact manifolds, see Chapter IV in 17
} 


\section{Proof of the main Results}

Now we arrive at

Proof of Theorem A. Let $f$ be a d-closed form in $\mathcal{E}^{p, q}(X)$ such that $[f] \in P^{p, q}(X)$. We like to prove that $Q([f],[f]) \geq 0$. Let $v$ be the smooth $(p-1, q-1)$-form given by Proposition 2.4. Then we have

$$
\left(f-\mathrm{dd}^{\mathrm{c}} v\right) \wedge \Omega \wedge \omega_{n-p-q+1}=0 .
$$

When either $p=0$ or $q=0$ we replace $\mathrm{dd}^{\mathrm{c}} v$ by 0 . In virtue of the identity (3.1), we are able to apply Proposition 2.1(b) to every point of $X$. Consequently, after an integration on $X$, we obtain that

$$
i^{p-q}(-1)^{\frac{(n-p-q)(n-p-q-1)}{2}} \int_{X}\left(f-\mathrm{dd}^{\mathrm{c}} v\right) \wedge\left(\bar{f}-\mathrm{dd}^{\mathrm{c}} \bar{v}\right) \wedge \Omega \geq 0 .
$$

Applying Stokes' Theorem to the left-hand side of the last line yields that

$$
\int_{X} f \wedge \bar{f} \wedge \Omega=\int_{X}\left(f-\operatorname{dd}^{\mathrm{c}} v\right) \wedge\left(\bar{f}-\operatorname{dd}^{\mathrm{c}} \bar{v}\right) \wedge \Omega .
$$

This, combined with (3.2), implies that $Q([f],[f]) \geq 0$. The equality happens if and only if $f=\mathrm{dd}^{\mathrm{c}} v$, in other words, $[f]=0$. Hence, the proof of the theorem is complete.

Proof of Theorem B. Let $\omega_{n-p-q+1}$ be an arbitrary Kähler form. Since $\operatorname{dim} \mathcal{H}^{p, q}(X)=\operatorname{dim} \mathcal{H}^{n-q, n-p}(X)$, it is sufficient to show that $\tau$ is injective. To this end let $\alpha$ be a d-closed form in $\mathcal{E}^{p, q}(X)$ such that

$$
\tau([\alpha])=[\alpha] \wedge[\Omega]=0 \text { in } \mathcal{H}^{n-q, n-p}(X) .
$$

Then we have that $[\alpha] \in P^{p, q}(X)$ and $Q([\alpha],[\alpha])=0$. Applying Theorem A yields that $[\alpha]=0$. Hence, $\tau$ is injective.

Proof of Theorem C. Let $\phi: \mathcal{H}^{n-q, n-p}(X) \longrightarrow \mathcal{H}^{n-q+1, n-p+1}(X)$ be given by

$$
\phi([\alpha]):=\left[\omega_{n-p-q+1}\right] \wedge[\alpha], \quad[\alpha] \in \mathcal{H}^{n-q, n-p}(X) .
$$

Theorem B implies that $\operatorname{dim} P^{p, q}(X)=\operatorname{dim} \operatorname{Ker} \phi$. On the other hand, by the classical Hodge theory (see [2, 6, 16, 17, ) we know that $\phi$ is surjective. Hence,

$$
\begin{aligned}
\operatorname{dim} \operatorname{Ker} \phi & =\operatorname{dim} \mathcal{H}^{n-q, n-p}(X)-\operatorname{dim} \mathcal{H}^{n-q+1, n-p+1}(X) \\
& =\operatorname{dim} \mathcal{H}^{p, q}(X)-\operatorname{dim} \mathcal{H}^{p-1, q-1}(X) .
\end{aligned}
$$

Consequently,

$$
\operatorname{dim} P^{p, q}(X)+\operatorname{dim} \mathcal{H}^{p-1, q-1}(X)=\operatorname{dim} \mathcal{H}^{p, q}(X) .
$$

On the other hand, it follows from Theorem B that the multiplication by $\left[\omega_{n-p-q+1}\right]$ is injective on $\mathcal{H}^{p-1, q-1}(X)$ and

$$
P^{p, q}(X) \cap\left[\omega_{n-p-q+1}\right] \wedge \mathcal{H}^{p-1, q-1}(X)=\{0\} .
$$

Hence, the desired decomposition follows from (3.3) and (3.4). 


\section{Another version of the Hodge-Riemann theorem}

In this section we describe the domain of validity of the mixed Hodge-Riemann theorem in the case where $p=q=1$. This problem is motivated by the dynamical study of holomorphic automorphisms on compact Kähler manifolds. An application of the mixed Hodge-Riemann theorem was given in the joint work of the first author and Nessim Sibony [4] (see also [5]). In order to present the results we need to introduce some notation.

Let $X$ be as usual a compact Kähler manifold of dimension $n$. Define

$$
\mathcal{H}^{p, p}(X, \mathbb{R}):=\mathcal{H}^{p, p}(X) \cap \mathcal{H}^{2 p}(X, \mathbb{R}) .
$$

Let $\mathcal{K}_{p}$ be the cone of all classes of smooth strictly positive $(p, p)$-forms in $\mathcal{H}^{p, p}(X, \mathbb{R})$. This cone is open and satisfies $-\overline{\mathcal{K}}_{p} \cap \overline{\mathcal{K}}_{p}=\{0\}$, where $\overline{\mathcal{K}}_{p}$ is the closure of $\mathcal{K}_{p}$. Each class in $\overline{\mathcal{K}}_{p}$ can be represented by a positive closed $(p, p)$-current. The cone $\mathcal{K}_{1}$ is the Kähler cone of $X$. Here, positivity of forms and currents of higher bidegree can be understood in the weak or strong sense. We refer to [3] for the basics on the theory of positive closed currents.

Fix a Kähler form $\omega$. Define $P^{p, q}(X)$ and $Q(\cdot, \cdot)$ as in (1.1) and (1.2) but for an arbitrary non-zero class $[\Omega]$ in $\overline{\mathcal{K}}_{n-p-q}$ and for $\omega_{n-p-q+1}:=\omega$. The class $[\Omega] \wedge[\omega]$ does not vanish since it can be represented by a non-zero positive closed current. Let $\mathcal{K}_{n-p-q}^{H R}$ be the cone of all classes $[\Omega] \in \mathcal{K}_{n-p-q}$ which satisfy the mixed HodgeRiemann Theorem (Theorem A), that is, $Q(\cdot, \cdot)$ is positive definite on $\mathcal{P}^{p, q}(X)$.

From now on we consider the case where $p=q=1$. The Poincaré duality implies that $\mathcal{P}^{1,1}(X)$ is a hyperplane of $\mathcal{H}^{1,1}(X)$ which depends continuously on $[\Omega]$. It follows by continuity that $\mathcal{K}_{n-2}^{H R}$ is an open cone in $\mathcal{H}^{n-2, n-2}(X, \mathbb{R})$. Theorem $\mathrm{A}$ implies that one of the connected components of $\mathcal{K}_{n-2}^{H R}$ contains all the products of $(n-2)$ Kähler classes. Observe that $\mathcal{P}^{1,1}(X)$ does not intersect $\mathcal{K}_{1}$ since $[\Omega]$ is the class of a positive closed current.

Let $\mathcal{L}_{n-2}$ be the set of all classes $[\Omega]$ in $\mathcal{H}^{n-2, n-2}(X, \mathbb{R})$ such that the wedge product map $[\alpha] \mapsto[\alpha] \wedge[\Omega]$ does not induce an isomorphism between $\mathcal{H}^{1,1}(X)$ and $\mathcal{H}^{n-1, n-1}(X)$. Observe that $\mathcal{L}_{n-2}$ is an algebraic cone defined by a homogeneous polynomial of degree $\operatorname{dim}_{\mathbb{C}} \mathcal{H}^{1,1}(X)$.

Proposition 4.1. The cone $\mathcal{K}_{n-2}^{H R}$ is a union of connected components of $\mathcal{K}_{n-2} \backslash \mathcal{L}_{n-2}$. In particular, it does not depend on the Kähler form $\omega$. Moreover, if $[\Omega]$ is a class in $\overline{\mathcal{K}}_{n-2}^{H R}$ then $Q(\cdot, \cdot)$ is positive semi-definite on $\mathcal{P}^{1,1}(X)$ and for $c \in \mathcal{P}^{1,1}(X)$ we have $Q(c, c)=0$ if and only if $c \wedge[\Omega]=0$.

Proof. It is clear that $\mathcal{L}_{n-2} \cap \mathcal{K}_{n-2}^{H R}=\varnothing$. Let $[\Omega]$ be a class in $\mathcal{K}_{n-2}$ which belongs to the boundary of $\mathcal{K}_{n-2}^{H R}$. We have to show that $[\Omega] \in \mathcal{L}_{n-2}$. By continuity, $Q(\cdot, \cdot)$ is positive semi-definite on $\mathcal{P}^{1,1}(X)$. Since $[\Omega] \notin \mathcal{K}_{n-2}^{H R}$, there exists $c \in \mathcal{P}^{1,1}(X), c \neq 0$, such that $Q(c, c)=0$. The Cauchy-Schwarz inequality implies that $Q\left(c, c^{\prime}\right)=0$ for every $c^{\prime}$ in the hyperplane $\mathcal{P}^{1,1}(X)$. We have seen that $[\omega]$ does not belong to $\mathcal{P}^{1,1}(X)$. On the other hand, $Q(c,[\omega])=0$ because $c \in \mathcal{P}^{1,1}(X)$. Consequently, $Q(c, \cdot)=0$. Therefore, the Poincaré duality implies that $c \wedge[\Omega]=0$. Hence, 
$[\Omega] \in \mathcal{L}_{n-2}$. The first part of the proposition is proved. We obtain the second part in the same way.

Remarks 4.2. Observe that $Q(\cdot, \cdot)$ is positive definite on $\mathcal{H}^{2,0}(X) \oplus \mathcal{H}^{0,2}(X)$ for $\Omega \in \mathcal{K}_{n-2}$. Then if $[\Omega] \in \mathcal{K}_{n-2}^{H R}$, the multiplication by $[\Omega]$ induces an isomorphism between $\mathcal{H}^{2}(X)$ and $\mathcal{H}^{n-2}(X)$.

Let $\widetilde{\mathcal{K}}_{n-2}$ be the cone of the classes in $\mathcal{H}^{n-2, n-2}(X, \mathbb{R})$ of all positive closed currents of bidegree $(n-2, n-2)$. This cone is convex and closed. Moreover, it contains $\mathcal{K}_{n-2}$ and satisfies $-\widetilde{\mathcal{K}}_{n-2} \cap \widetilde{\mathcal{K}}_{n-2}=\{0\}$. Let $\widetilde{\mathcal{K}}_{n-2}^{H R}$ denote the cone of all classes in $\widetilde{\mathcal{K}}_{n-2}$ which satisfy the mixed Hodge-Riemann theorem. Then Proposition 4.1 holds for $\widetilde{\mathcal{K}}_{n-2}^{H R}$. More precisely, $\widetilde{\mathcal{K}}_{n-2}^{H R}$ is a union of connected components of $\widetilde{\mathcal{K}}_{n-2} \backslash \mathcal{L}_{n-2}$.

The following type of results might be useful in the dynamical study of holomorphic automorphisms (see [4, 5])

Corollary 4.3. Let $c_{1}, \ldots, c_{n-2}$ be classes of $\overline{\mathcal{K}}_{1}$ and let $c_{n-1}$ be a Kähler class. Then a class $c$ in $\mathcal{H}^{1,1}(X)$ satisfies $c \wedge c_{1} \wedge \cdots \wedge c_{n-1}=0$ and $c \wedge c \wedge c_{1} \wedge \cdots \wedge c_{n-2}=0$ if and only if $c \wedge c_{1} \wedge \cdots \wedge c_{n-2}=0$.

Proof. Since each $c_{i}$ can be approximated by Kähler forms, Theorem A implies that $[\Omega]:=c_{1} \wedge \cdots \wedge c_{n-2}$ belongs to $\overline{\mathcal{K}}_{n-2}^{H R}$. Therefore, it is sufficient to apply Proposition 4.1 .

\section{REFERENCES}

[1] B. Berndtsson and N. Sibony, The $\bar{\partial}$-equation on a positive current, Invent. Math., 147(2), (2002), 371-428.

[2] J. Bertin, J.-P. Demailly, L. Illusie and C. Peters, Introduction à la théorie de Hodge, Panoramas et Synthèses, 3, Société Mathématique de France, Paris, 1996. vi+273 pp.

[3] J.-P. Demailly, Complex analytic and algebraic geometry, available at www-fourier.ujf-grenoble.fr/ demailly.

[4] T.-C. Dinh and N. Sibony, Groupes commutatifs d'automorphismes d'une variété kählérienne compacte, Duke Math. J., 123(2), (2004), 311-328.

[5] T.-C. Dinh and N. Sibony, Green current for holomorphic automorphisms of compact Kähler manifolds, J. Amer. Math. Soc., 18, (2005), 291-312.

[6] Ph. Griffiths and J. Harris, Principles of algebraic geometry, Reprint of the 1978 original. Wiley Classics Library. John Wiley \& Sons, Inc., New York, 1994. xiv+813 pp.

[7] M. Gromov, Convex sets and Kähler manifolds, Advances in differential geometry and topology, 1-38, World Sci. Publishing, Teaneck, NJ, 1990.

[8] A. G. Khovanskii, Newton polyhedra, and the genus of complete intersections, (Russian), Funktsional. Anal. i Prilozhen., 12(1), (1978), 51-61.

[9] A. G. Khovanskii, The geometry of convex polyhedra and algebraic geometry, (Russian), Uspehi Mat. Nauk., 34(4), (1979), 160-161.

[10] A. G. Khovanskii, Algebra and mixed volumes, in: Y. D. Burago and V. A. Zalgaller, Geometrical Inequalities, Series of Geometry, Vol. 285, Springer-Verlag, Berlin-New York, 1988, 182-207.

[11] P. McMullen, On simple polytopes, Invent. Math., 113(2), (1993), 419-444.

[12] B. Teissier, Appendix: Sur une inégalité à la Minkowski pour les multiplicités, in: D. Eisenbud and $\mathrm{H}$. Levine, An algebraic formula for the degree of a $\mathcal{C}^{\infty}$ map germ, Ann. Math., 106(1), (1977), 19-44. 
[13] B. Teissier, Du théorème de l'index de Hodge aux inégalités isopérimétriques, C. R. Acad. Sci. Paris Sér. A-B, 288(4), (1979), A287-A289.

[14] B. Teissier, Variétés toriques et polytopes, (French), Bourbaki Seminar, Vol. 1980/81, 71-84, Lecture Notes in Math., 901, Springer, Berlin-New York, 1981.

[15] V. A. Timorin, Mixed Hodge-Riemann bilinear relations in a linear context, Funct. Anal. Appl., 32(4), (1998), 268-272.

[16] C. Voisin, Théorie de Hodge et géométrie algébrique complexe, Cours Spécialisés, 10, Société Mathématique de France, Paris, 2002. viii+595 pp.

[17] R. O. Wells, Differential analysis on complex manifolds, Second edition. Graduate Texts in Mathematics, 65, Springer-Verlag, New York-Berlin, 1980. x+260 pp.

Tien-Cuong Dinh, Mathématique-Bât 425, UMR 8628, Université Paris-Sud F91405 Orsay, France

E-mail address: TienCuong.Dinh@math.u-psud.fr

Viêt-Anh Nguyên, MaX-Planck Institut für Mathematik, Vivatsgasse 7, D-53111, Bonn, Germany

E-mail address: vietanh@mpim-bonn.mpg.de 